\title{
What Would Dr. James Parkinson Think Today? II. Neuroimaging in Parkinson's Disease
}

\author{
Roger L. Albin, MD ${ }^{1,2,3,4 *}$ \\ ${ }^{1}$ Department of Neurology, University of Michigan, Ann Arbor, Michigan, USA \\ ${ }^{2}$ Neurology Service \& Geriatrics Research, Education, and Clinical Center, Veterans Affairs Ann Arbor Health System, Ann Arbor, Michigan, USA \\ ${ }^{3}$ University of Michigan Morris K. Udall Center of Excellence for Parkinson's Disease Research, \\ Ann Arbor, Michigan, USA \\ ${ }^{4}$ University of Michigan Alzheimer Disease Center, Ann Arbor, Michigan, USA
}

Dr. James Parkinson would be impressed and gratified by the progress made through the application of neuroimaging methods in Parkinson disease (PD). Parkinson was considerably more than an astute and articulate clinician. Parkinson epitomizes the productive scientists (a term coined by the English polymath William Whewell in 1833) of his time. The great majority of his scientific contemporaries were essentially amateurs, either wealthy men such as the great naturalist Joseph Banks, or middling class professionals, often physicians or clergy, engaged in occupations that provided enough income and leisure time to pursue intellectual interests. In the expanding public sphere of Enlightenment Europe, physicians such as Parkinson, Edward Jenner, and John Hunter were active participants in broad networks of correspondence, social clubs, and learned societies focused on improving human knowledge. In addition to his significant contributions to medicine, Parkinson was a pioneering paleontologist and one of the founders of British geoscience.

Parkinson's famous essay demonstrates his understanding of the limitations of clinical observation and the need for more definitive methods: "it is acknowledged, that mere conjecture takes the place of experiment; and, that analogy is the substitute for anatomical explanation, the only sure foundation for pathological knowledge." 1 In his time, "anatomical

*Correspondence to: Dr. Roger L. Albin, 5023 BSRB, 109 Zina Pitcher Place, Ann Arbor, MI, USA, 48109-2200; ralbin@umich.edu

Funding agencies: Supported by R56 NS082941 and P50NS091856.

Relevant conflicts of interest/financial disclosures: R.L.A. serves on the editorial boards of Neurology, Annals of Neurology, Experimental Neurology, and Neurobiology of Disease. He serves on the Data Safety and Monitoring Boards for the Laquinimod in Huntington disease trial (LEGATO-HD), (Icon-Teva) and lonis 443139 trials. He has grant support from NIH-NINDS and the Michael J. Fox Foundation.

Received: 15 January 2017; Accepted: 16 January 2017

Published online in Wiley Online Library

(wileyonlinelibrary.com) DOI: 10.1002/mds.26951 explanation" was largely limited to the visual observation of gross pathologies. Parkinson used the best available knowledge of his time to speculate about the sites of pathology in PD: "A diseased state of the medulla spinalis, in that part which is contained in the canal, formed by the superior cervical vertebrae, and extending, as the disease proceeds, to the medulla oblongata." Parkinson inferred correctly that the distinctive features of PD were different from those resulting from peripheral nervous system pathologies. His observations of initial limb, particularly arm, involvement undoubtedly directed his attention to the cervical spinal cord. His description of bulbar dysfunction in more advanced PD prompted his suggestion of the progression of pathology to the medulla, a concept that anticipates modern models of spreading CNS pathology in PD.

Parkinson practiced when clinico-pathologic correlation methods were pioneered by his contemporaries John Hunter and Marie Francois Xavier Bichat. In a recurring theme of the history of medicine, technological improvements expanded the power of clinico-pathologic correlation. In the 19th century, improvements in microscope technology and the development of histologic methods completely transformed understanding of disease. Imaging technologies have had an analogous impact in our time. Several papers in this issue of Movement Disorders illustrate the power of contemporary imaging methods. Strafella and colleagues ${ }^{2}$ nicely review the impact of molecular (positron emission tomography) imaging on PD research. In a remarkable extension of the clinico-pathologic correlation method, molecular imaging methods allow in vivo identification of key brain changes underlying specific features of PD. Neuroimaging methods allow other forms of important investigations in $\mathrm{PD}$, such as expanding clinical pharmacology and development of accurate biomarkers. Politis and colleagues ${ }^{3}$ describe an interesting study in which molecular imaging was used to probe a key pharmacokinetic variable, striatal 
extracellular dopamine levels, during intestinal Ldopa infusions. Langley and colleagues ${ }^{4}$ describe a novel MRI analysis approach to measure a possible marker of dopaminergic neuron degeneration, substantia nigra pars compacta iron deposition.

In addition to identifying crucial sites of pathology in PD in vivo, modern imaging methods directly address other important issues raised by Parkinson. Parkinson was acutely aware of the need to differentiate PD from other diseases. Although the discovery of PD mimics such as progressive supranuclear palsy, multiple system atrophy, and corticobasal syndrome lay decades in the future, Parkinson devoted chapter III of An Essay on the Shaking Palsy ("Shaking Palsy Distinguished From Other Diseases With Which It May Be Confounded") to clearly establishing the distinctive features of PD. Parkinson's discussion in this chapter demonstrates his awareness that the accurate identification of disease is necessary to understand pathogenetic mechanisms. Parkinson was also clearly aware of the importance of studying early phases of disease pathogenesis and pathophysiology:

It seldom happens that the agitation extends beyond the arms within the first two years; which period, therefore, if we were disposed to divide the disease into stages, might be said to comprise the first stage. In this period, it is very probable, that remedial means might be employed with success.

Parkinson would probably be impressed with the ability of imaging methods to interrogate early brain changes in PD, which he would recognize as crucial to developing disease-modifying therapies.

\section{What Would Dr. Parkinson Think Today?}

Although Parkinson would be impressed by results of neuroimaging studies of PD, and perhaps startled by the power of these methods, the underlying intellectual principles would be familiar. Parkinson was a typical figure of the Enlightenment, and his achievements illustrate one of the great themes of the Enlightenment, the concept of useful knowledge. Inspired by the success of Newtonian physics, Enlightenment intellectuals believed that the underlying structure of the universe was discoverable through the exercise of human reason and that this knowledge could be used for the benefit of humanity. ${ }^{5-7}$ In Parkinson's time, these were novel and contested ideas. Parkinson would probably regard our present, albeit incomplete, understanding of PD as vindication of his ideals.

\section{References}

1. Parkinson J. An essay on the shaking palsy. Project Gutenberg. https://www.gutenberg.org/ebooks/23777. Accessed January 13, 2017.

2. Strafella A, Bohnen N, Perlmutter J, et al. Molecular imaging to track Parkinson's disease and atypical parkinsonisms: new imaging frontiers. Mov Dis 2017;31(2).

3. Politis M, Sauerbier A, Loane C, et al. Sustained striatal dopamine levels following intestinal levodopa infusions in patients with PD. Mov Dis 2017;31(2).

4. Langley J, Huddleston D, Sedlacik J, Boelmans K, Hu X. Parkinson's disease related increase of $\mathrm{T}_{2}^{*}$ weighted hypointensity in substantia nigra pars compacta. Mov Dis 2017;31(2).

5. Jacob MC. Scientific Culture and the Making of the Industrial West. Oxford, UK: Oxford University Press, 1997.

6. Cassirer E. The Philosophy of the Enlightenment. Princeton, NJ: Princeton University Press, 2009.

7. Mokyr J. The Enlightened Economy: An Economic History of Britain 1700-1850. Princeton, NJ: Princeton University Press, 2012. 